\title{
BMJ Open Feasibility of improving identification of familial hypercholesterolaemia in general practice: intervention development study
}

\author{
Nadeem Qureshi, ${ }^{1}$ Stephen Weng, ${ }^{1}$ Jennifer Tranter, ${ }^{1}$ Alia El-Kadiki, ${ }^{2}$ Joe Kai ${ }^{1}$
}

To cite: Qureshi N, Weng S, Tranter $\mathrm{J}$, et al. Feasibility of improving identification of familial

hypercholesterolaemia in general practice: intervention development study. BMJ Open 2016;6: e011734. doi:10.1136/ bmjopen-2016-011734

- Prepublication history for this paper is available online. To view these files please visit the journal online (http://dx.doi.org/10.1136/ bmjopen-2016-011734).

Received 1 March 2016 Revised 15 April 2016 Accepted 3 May 2016

CrossMark

${ }^{1}$ Division of Primary Care, NIHR School of Primary Care Research, University of Nottingham, Nottingham, UK ${ }^{2}$ Nottingham University Hospitals NHS Trust, Nottingham, UK

Correspondence to Professor Nadeem Qureshi; nadeem.qureshi@nottingham. ac.uk

\section{ABSTRACT}

Objectives: To assess the feasibility of improving identification of familial hypercholesterolaemia $(\mathrm{FH})$ in primary care, and of collecting outcome measures to inform a future trial.

Design: Feasibility intervention study.

Setting: 6 general practices (GPS) in central England. Participants: 831 eligible patients with elevated cholesterol $>7.5 \mathrm{mmol} / \mathrm{L}$ were identified, by search of electronic health records, for recruitment to the intervention.

Intervention: Educational session in practice; use of opportunistic computer reminders in consultations or universal postal invitation over 6 months to eligible patients invited to complete a family history questionnaire. Those fulfilling the Simon-Broome criteria for possible FH were invited for GP assessment and referred for specialist definitive diagnosis.

Outcome measures: Rates of recruitment of eligible patients, identification of patients with possible $\mathrm{FH}$, referral to specialist care, diagnosis of confirmed $\mathrm{FH}$ in specialist care; and feasibility of collecting relevant outcome measures for a future trial.

Results: of 173 general practices, 18 were interested in participating and 6 were recruited. From 831 eligible patients, 127 (15.3\%) were recruited and completed family history questionnaires: $86(10.7 \%)$ through postal invitation and 41 (4.9\%) opportunistically. Among the 127 patients, 32 (25.6\%) had a possible diagnosis of FH in primary care. Within 6 months of completing recruitment, 7 patients had had specialist assessment confirming 2 patients with definite $\mathrm{FH}$ (28.6\%), and 5 patients with possible FH $(71.4 \%)$. Potential trial outcome measures for lipid tests, statin prescribing and secondary causes of hypercholesterolaemia were extracted using automated data extraction from electronic records alone without recourse to other methods.

Conclusions: The intervention is feasible to implement in GP, and facilitates recruitment of patients with raised cholesterol for targeted assessment and identification of $\mathrm{FH}$. Extracting data directly from electronic records could be used to evaluate relevant outcome measures in a future trial.
Strengths and limitations of this study

- This feasibility study was able to engage general practices (GPS) and patients from underserved populations in an intervention to identify familial hypercholesterolaemia (FH) more systematically.

- Extraction of data using automated GP computer searches can capture important outcome measures for a future trial of FH identification.

- Further strategies are needed if engaging eligible patients is to be improved on opportunistic contact during GP consultations.

- The 6-month patient follow-up period used was too short to elicit complete data on all relevant outcome measures, such as eventual specialist assessment.

\section{INTRODUCTION}

Familial hypercholesterolaemia $(\mathrm{FH})$ is one of the most common inherited autosomal dominant disorders and is associated with elevated low-density lipoprotein cholesterol levels. In the UK, around 1 in 500 to 1 in 200 people are affected by the heterozygote form of this condition. ${ }^{1}$ Left untreated this can lead to premature coronary heart disease in those individuals affected. ${ }^{2}{ }^{3}$ However, with appropriate lipid-lowering treatment, intervention is highly effective and life expectancy can return to normal. ${ }^{4}$

Despite the overwhelming case for treatment and national guidelines recommending early identification, it is estimated that up to $80 \%$ of heterozygote $\mathrm{FH}$ still remain unrecognised. ${ }^{5}{ }^{6}$ Of most concern, individuals with raised cholesterol levels documented in general practice (GP) medical records may not be recognised to have possible $\mathrm{FH}$. However, primary care is an ideal setting to identify possible $\mathrm{FH}$ cases through identification of those with raised cholesterol and relevant family histories. Current UK National Institute for Health and Care Excellence 
Box 1 Referral criteria for diagnosis: abridged SimonBroome diagnostic criteria

In adults, total cholesterol $>7.5 \mathrm{mmol} / \mathrm{L}$ and low density lipoprotein $>4.9 \mathrm{mmol} / \mathrm{L}$.

- Plus for a diagnosis of possible familial hypercholesterolaemia (FH), family history of myocardial infarction at age $<60$ years in first-degree relative, age $<50$ years in second-degree relative, or a family history of raised cholesterol levels.

- Plus for a diagnosis of definite familial hypercholesterolaemia, tendon xanthomata in patient or in first-degree or seconddegree relative.

- Patients with 'definite' or 'possible' familial hypercholesterolaemia should be referred to a specialist with expertise in familial hypercholesterolaemia, to confirm the diagnosis, management and coordination of the testing of relatives.

(NICE) guidelines recommend that these patients are identified using Simon-Broome criteria (box 1). ${ }^{67}$

Using these criteria, a positive family history is based on patients recalling premature coronary heart disease or raised cholesterol levels in their first-degree and/or second-degree relatives. This level of detail is absent or poorly recorded in GP electronic health records (EHRs), ${ }^{8}$ so further work to collate the family history is required, such as using a validated self-administered family history questionnaire. ${ }^{9-12}$ Using a combination of searching GP EHRs for patients with raised cholesterol, with completion of a family history questionnaire, individuals with possible FH may be more actively and appropriately identified in primary care.

In line with Medical Research Council guidelines, ${ }^{13}$ this feasibility study aimed to inform the development of an intervention to identify $\mathrm{FH}$ more proactively in primary care. We investigated if the approaches used, study procedures and analysis were feasible, or might be enhanced, prior to their use in a future randomised controlled trial (RCT). ${ }^{13}$ We explored patients being targeted and invited for further assessment of possible $\mathrm{FH}$, opportunistically when they consult, using computerised patientspecific reminders when their serum cholesterol is above diagnostic threshold $(7.5 \mathrm{mmol} / \mathrm{L})$ and was recorded in electronic medical records. ${ }^{14}{ }^{15}$ In addition, we explored an approach of practices simply mailing all patients with a cholesterol level ever recorded above diagnostic threshold. The specific objectives were to gain experience of:

- patient recruitment, identification, referral and diagnosis rates

- using opportunistic and universal postal recruitment strategies

- whether relevant outcome measures for a proposed trial may be extracted from automated searches of GP EHRs.

\section{METHODS}

Study design

This was a feasibility study with process evaluation to inform a RCT. The study duration was 17 months, ending in August 2015, and was approved by NRES Committee West Midlands-Solihull (Reference 12/WM/0322).

\section{Participants}

All 173 general practices in Nottinghamshire were invited to participate in the study through the local Primary Care Clinical Research Network, and we received expressions of interest from 18 practices. Further information was given to all 18 practices, and the first six eligible practices which responded, were recruited to the study (4 inner city practices, 1 suburban and 1 rural practice).

Eligible patients were aged 18 years or over at the start of the study in the recruited general practices with a previous record, in the computer medical records, of cholesterol at a level $>7.5 \mathrm{mmol} / \mathrm{L}$. Those who already had a confirmed diagnosis of familial hypercholesterolaemia were excluded.

\section{Intervention and procedure}

The intervention began with a one-hour educational session at each recruited practice. This involved an update session on the identification and investigation of FH in line with current NICE guidelines, ${ }^{7}$ and linked to Simon-Broome criteria. ${ }^{6}$ General practitioners were familiarised with the proposed computer-based alert message which appears when eligible patients' medical records are accessed. They were also given a laminated sheet to use when eligible patients consulted their general practitioners as an aide memoire for further assessment. This outlined the Simon-Broome criteria, and a prompt to investigate and exclude secondary causes of hypercholesterolaemia such as diabetes, hypothyroidism, chronic kidney disease and liver disease.

Following training, a baseline computer data extraction was completed which identified 831 eligible patients, with cholesterol levels above $7.5 \mathrm{mmol} / \mathrm{L}$ from the six practices. These patients' EHRs were tagged with an alert message to check for secondary causes of elevated cholesterol, invite the patient to participate in the study, repeat the lipid profile and manage in line with NICE guidelines/Simon-Broome criteria for $\mathrm{FH}^{7}$ This alert appeared when the individual's computer records were accessed. This opportunistic approach was continued for 6 months. Four months into the intervention, eligible patients identified at baseline who had not already been invited opportunistically, were all approached through a postal invitation from their practice.

Eligible patients were given study packs by their general practitioners opportunistically, or had them mailed to them by their practice. These included study information/consent and blood test forms to have a follow-up fasting lipid profile (comprising total cholesterol, LDL cholesterol and triglyceride) unless lipid profile was done in the last 6 months, a Family History and Symptom Questionnaire (FHSQ) seeking details on family history of myocardial infarction and cholesterol, together with photos to identify tendon xanthoma in 
Achilles tendons and hands. The consent form and FHSQ were returned to the research team office, with recruited patients' participation in the study using a computer recruitment code tagged onto the patient's GP EHRs. The research team then collated information on fasting lipid results, current stain therapy, family history and reported any possibility of FH diagnosis, in line with Simon-Broome criteria (box 1) back to GPs to facilitate contacting participants. Participants fulfilling SimonBroome criteria for possible $\mathrm{FH}$ were asked to arrange an appointment with their general practitioner. The general practitioners were advised to examine patients for signs of FH, exclude secondary causes of raised cholesterol, and refer those patients who met Simon-Broome criteria to the local lipid specialist clinic. Those not fulfilling FH diagnosis based on Simon-Broome criteria were reassured, and a healthy living leaflet provided.

\section{Outcome measurements}

The primary measures assessed the feasibility of this intervention (see box 2). Recruitment rate was

\section{Box 2 FAMCHOL feasibility study outcome measures} assessed

Primary measures

- Recruitment rate;

- Referral rate;

- Diagnosis rate:

- Possible familial hypercholesterolaemia (FH) identified primary care,

- Possible FH confirmed by secondary care,

- Definite FH confirmed by secondary care;

- Retention rate;

Extracting relevant outcome measures for proposed trial

- Cholesterol level:

- Latest total cholesterol levels ( $\mathrm{mmol} / \mathrm{L})$,

- Latest low density lipoprotein (LDL) cholesterol levels (mmol/L),

- Proportion of patients who have a total cholesterol $\geq 7.5 \mathrm{mmol} / \mathrm{L}$ or LDL-C $\geq 4.9 \mathrm{mmol} / \mathrm{L}$;

- Repeat cholesterol (lipid profile) test;

- Patients diagnosed with secondary cause (diabetes, hypothyroidism, chronic kidney disease, liver disease);

- Family history of coronary heart disease assessed;

- Quality of coronary heart disease family history assessed (age, condition, degree of relation);

- Thyroid stimulating hormone assessment (proxy for hypothyroidism investigations);

- HbA1c assessment (proxy for diabetes investigation);

- Serum creatinine assessment (proxy for chronic kidney disease investigations);

- Bilirubin, alkaline phosphatase (ALP) or glutamyl transpeptidase (gamma GT) assessment (proxy for liver disease);

- Arcus senilis or xanthalasma diagnosed;

- Proportion of patients prescribed statins;

- Proportion of patients prescribed high potency statins treatment (simvastatin $\geq 80 \mathrm{mg} /$ day; atorvastatin $\geq 20 \mathrm{mg} /$ day; rosuvastatin $\geq 10 \mathrm{mg} /$ day)

- Dietary or weight management advice;

Smoking cessation advice. determined during the 6 month study period, while referral, diagnosis and retention rate were assessed up to 6 months after completing recruitment (12 months after the start of the intervention). Diagnosis of confirmed FH in secondary care was based on clinical criteria (ie, Simon-Broome criteria). Relevant outcome measures for a proposed follow-up RCT were derived from the primary care recommendations in the NICE FH guidelines. This includes repeat testing for full lipid profile; excluding secondary causes of raised cholesterol and collecting comprehensive family history. The feasibility of extracting such data from GP EGRs was assessed using both an automated process via medical codes and a manual process in which a researcher reviewed patients' records, including free text entries and hospital letters. This was performed 6 months after the start of the intervention in each practice.

\section{Statistical analysis}

As a feasibility study, we assessed the feasibility of the intervention, recruitment process and study instruments; hence, a sample size for proposed outcome measure was not appropriate. All primary outcome measures (recruitment, referral, retention and diagnosis rates) were presented descriptively. For proposed trial outcome measures (NICE guideline compliance), a descriptive analysis presented numbers and percentages for all categorical variables; mean and SD for all continuous variables at 6-month follow-up. All analyses were conducted in STATA 14 MP4.

\section{RESULTS}

\section{Primary outcomes: feasibility of FAMCHOL study process} Recruitment rate

Figure 1 outlines the study recruitment process and procedure for the 831 eligible patients $(2.36 \%$ of total $)$ identified at baseline from 35438 patients, over 18 years, registered with the six GPs.

Opportunistically, out of 207 study packs handed out, 41 (19.8\% of study packs; $4.9 \%$ of all eligible patients) completed consent forms and Family History/Symptom Questionnaire. At 4 months, 802 study packs were mailed out to the outstanding eligible participants, and an additional 86 (10.7\% all mail-outs; $10.3 \%$ of all eligible patients) were recruited using this systematic approach. By the end of the 6-month recruitment process, 127 patients consented and were recruited to the study which gave an overall response and engagement rate of $15.3 \%$, (ranging from $7.9 \%$ to $15.8 \%$ at the four inner city practices, $13.9 \%$ at the suburban practice and $26.7 \%$ at the rural practice).

\section{Identification and referral rate}

Among the 127 consenting patients, 125 could be assessed for FH against Simon-Broome criteria. One patient declined follow-up blood lipids, and another 
Figure 1 FAMCHOL study procedure and process. $\mathrm{FH}$, familial hypercholesterolaemia; $\mathrm{GP}$, general practice.

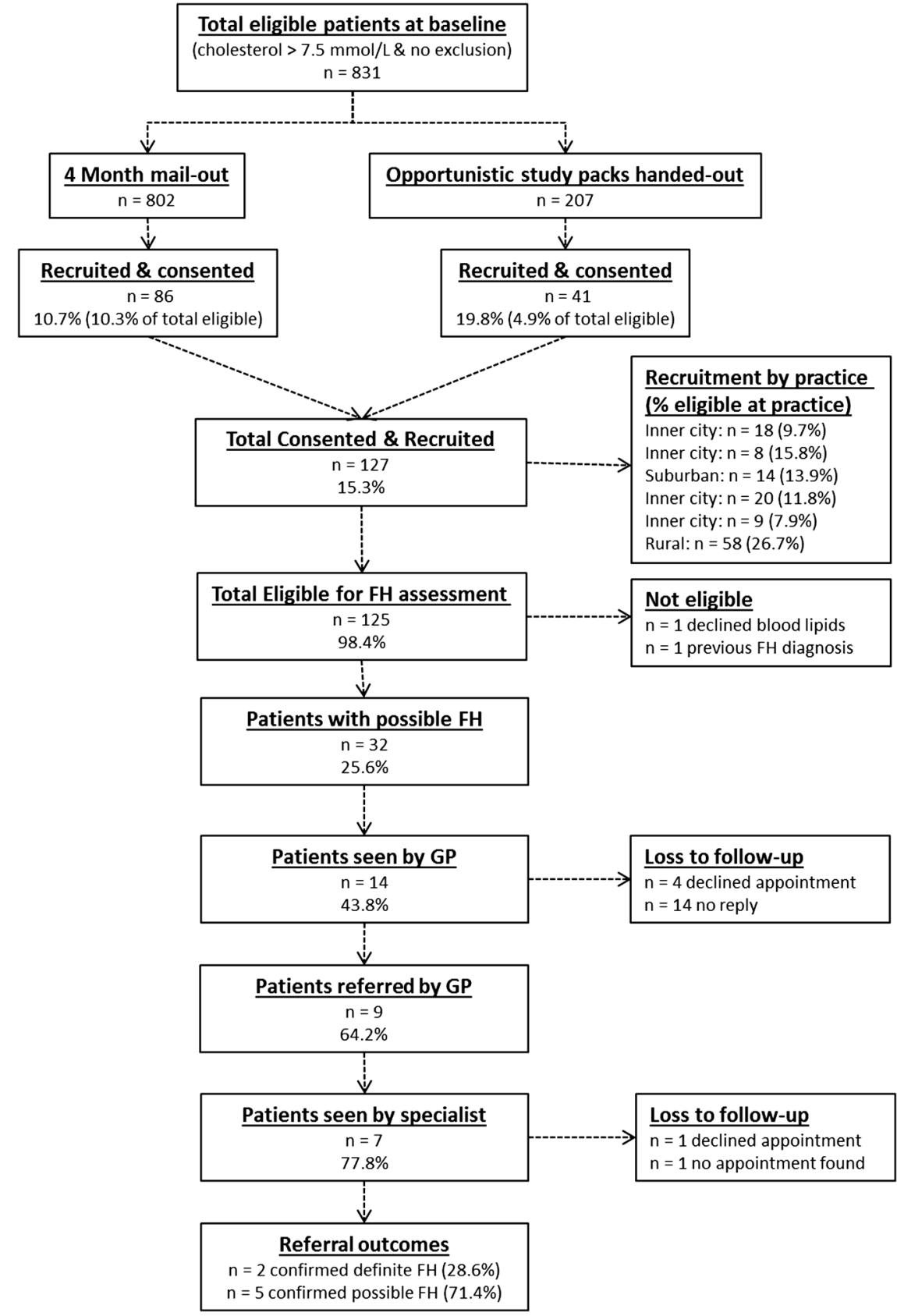

patient had previously been diagnosed with familial hypercholesterolaemia on more detailed review of previous medical records. Thirty-two $(25.6 \%$ of 125 eligible for assessment) patients had a possible diagnosis of $\mathrm{FH}$ and were invited to see their general practitioners. Fourteen patients $(43.8 \%$ of 32 with possible $\mathrm{FH})$ were actually seen by their general practitioner, while four patients declined the appointment and 14 patients did not respond to the invitation. Subsequently, nine patients $(64.2 \%$ of 14 seen by GPs) were referred to lipid specialist. Further, seven patients $(77.8 \%$ of the nine referred by GPs) were actually seen by specialists, as one patient declined the referral appointment and the other patient's referral could not be found in secondary care by the 12-month study period end.

\section{Diagnosis rate}

Of the seven patients with probable $\mathrm{FH}$ referred and seen by a specialist, two patients $(28.6 \%)$ were confirmed with definite $\mathrm{FH}$ (by specialist examination) and five patients $(71.4 \%)$ were confirmed with possible $\mathrm{FH}$ (mutation-negative for monogenic FH).

\section{Proposed trial outcomes: feasibility of data extraction}

From 127 participants recruited, data on clinical records from $118(95 \%)$ patients could be extracted from GP EHRs. Data from seven patients were excluded from the analysis due to transferring from practice. Table 1 details patient clinical characteristics based on the proposed outcome measures for 118 patients at 6 months from the start of the intervention through automated 
Table 1 Proposed outcome measures extracted from primary care computer records using an automated extraction at the end of the study period

\begin{tabular}{|c|c|c|}
\hline Patient outcome measures & Male $(n=46)$ & Female $(n=72)$ \\
\hline Age (years) & $58(9.0)$ & $56(7.5)$ \\
\hline Total cholesterol (mmol/L) & $5.8(1.5)$ & $6.4(1.4)$ \\
\hline LDL cholesterol (mmol/L) & $3.6(1.3)$ & $3.8(1.5)$ \\
\hline Number with latest TC $\geq 7.5 \mathrm{mmol} / \mathrm{L}$ or $\mathrm{LDL}-\mathrm{C} \geq 4.9 \mathrm{mmol} / \mathrm{L}(\%)$ & $12(26)$ & $23(32)$ \\
\hline Number with repeat cholesterol test within 6 months after study start date (\%) & $41(89)$ & $60(83)$ \\
\hline Number of diagnosed with secondary cause ${ }^{*}$ within 6 months after study start date (\%) & $5(11)$ & $5(7)$ \\
\hline $\begin{array}{l}\text { Number with any family history of coronary heart disease assessed within } 6 \text { months after } \\
\text { study start date (\%) }\end{array}$ & $14(30)$ & $31(43)$ \\
\hline $\begin{array}{l}\text { Number with any complete family history }+ \text { of coronary heart disease assessed within } \\
6 \text { months after study start date (\%) }\end{array}$ & $1(2)$ & $6(8)$ \\
\hline Number with TSH assessed within 6 months after study start date $\ddagger$ (\%) & $14(30)$ & $17(23)$ \\
\hline Number with $\mathrm{HbA} 1 \mathrm{c}$ assessed within 6 months after study start date $\S(\%)$ & $6(13)$ & $17(24)$ \\
\hline Number with serum creatinine assessed within 6 months after study start datef (\%) & $19(41)$ & $29(40)$ \\
\hline $\begin{array}{l}\text { Number with bilirubin, ALP or gamma GT assessed within } 6 \text { months after study start } \\
\text { date }^{\star \star}(\%)\end{array}$ & $19(41)$ & $22(31)$ \\
\hline $\begin{array}{l}\text { Number with arcus senilis or xanthalasma diagnosed within } 6 \text { months after study start } \\
\text { date (\%) }\end{array}$ & $0(0)$ & $0(0)$ \\
\hline Number of prescribed any statins within 6 months after study start date (\%) & $14(30)$ & $13(18)$ \\
\hline Number of prescribed high-potency statinst† within 6 months after study start date (\%) & $4(9)$ & $2(3)$ \\
\hline $\begin{array}{l}\text { Number of given dietary or weight management advice within } 6 \text { months after study start } \\
\text { date }(\%)\end{array}$ & $27(59)$ & $33(46)$ \\
\hline Number of given smoking cessation advice within 6 months after study start date (\%) & $19(41)$ & $15(21)$ \\
\hline 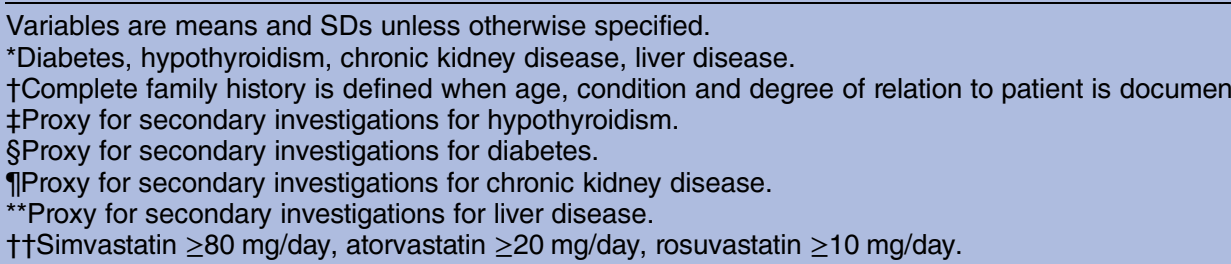 & & \\
\hline
\end{tabular}

extraction from GP EHRs. Although all patients had a cholesterol assessment completed, 3\% $(\mathrm{n}=4)$ of the sample did not have the LDL cholesterol measurement documented into their EHRs.

Among the 118 recruited patients, there were more men than women $(61 \%$ men, $39 \%$ women), with an average age being 58 years (SD 9.0) for men and 56 years (SD 7.5) for women. The latest mean total cholesterol was $5.8 \mathrm{mmol} / \mathrm{L}(\mathrm{SD} 1.5)$ and $6.4 \mathrm{mmol} / \mathrm{L}$ (SD 1.4) for men and women, respectively. The mean LDL cholesterol was $3.6 \mathrm{mmol} / \mathrm{L}$ (SD 1.3 ) and $3.8 \mathrm{mmol} / \mathrm{L}$ (SD 1.5) for men and women, respectively. Additionally, 12 men (26\%) and 23 women (32\%) were found to have an elevated total or LDL-cholesterol. All outcome measures extracted purely from automated process are presented in table 1.

Considering other measures, manual extraction only showed a small absolute increase in number of patients diagnosed with a secondary causes of hypercholesterolaemia (men: $+2 \%$; women $+3 \%$ ), statin prescribing (men: $+1 \%$; women $+4 \%$ ), and in smoking cessation advice given (men: $+2 \%$; women: $+8 \%$ ). The low level of family history recording, particularly when identifying a 'complete family history', was only slightly improved by an absolute increase of $5 \%$ for both men and women
(+2 men; +4 women) when manually extracting free text in the GP EHRs. However, in manual extraction, GPs arranged and documented more investigations for secondary causes (thyroid stimulating hormone: $+30 \%$ for men; $+36 \%$ for women; HbAlc: $+18 \%$ for men, $+33 \%$ for women; serum creatinine: $+55 \%$ for men, $+56 \%$ for women; liver function test: $+52 \%$ for men, $+58 \%$ for women), and dietary or weight management advice given (men: $+12 \%$; women: $+20 \%$ ). In automated computer data extraction, clinical features of $\mathrm{FH}$ (arcus senilis or xanthalasma) were not recorded, while in manual search, $7 \%$ (1 man; 7 women) of all participants had arcus senilis or tendon xanthalasma documented in free text.

\section{DISCUSSION}

This study showed that it is feasible to engage and recruit patients with raised cholesterol for more systematic identification of familial hypercholesterolaemia in primary care, both through opportunistic GP contact and by postal invitation. Although postal invitation resulted in more eligible patients being recruited than opportunistic invitation, the latter had double the response rate. This suggests both strategies should be 
adopted for patient recruitment in any future study. While only a feasibility study, new cases of definite FH were identified in the current work who are now on appropriate treatment and management pathways to reduce their previously elevated cardiovascular risk.

By using automated GP computer searches, we were able to capture relevant outcome measures for a proposed trial on lipid testing, statin prescribing and possible secondary causes of hypercholesterolaemia, without burdening patients and clinicians with data collection forms. This could potentially improve collating such outcome measures in this and other studies.

\section{Strengths and limitations}

The majority of practices recruited to this study (four out of six) were from socially disadvantaged inner city populations where prior identification rates of $\mathrm{FH}$ were likely to be lower. Although one affluent rural practice accounted for nearly half the recruited participants, the approaches used successfully recruited patients from practices with underserved populations. In addition, successful extraction of outcome data from GP health records was achieved from 95\% (118) of recruited patients using automated searches.

Owing to the pragmatic nature of this study, some patients may have received the recruitment packs twice (opportunistic and systematic). However, for ethical reasons, we could not identify patients given recruitment packs opportunistically, and did not consent to participate in the study. In terms of identifying eligible patients, we recognised that eligibility criteria did not consider the effects of statin treatment. There were individuals who had cholesterol levels below the $7.5 \mathrm{mmol} / \mathrm{L}$ threshold who were already on statins at the time of recording highest cholesterol level in the electronic medical records. If these treatment effects were considered, ${ }^{16}$ there may be more eligible patients than we previously thought. Additionally, recruitment of eligible patients to the study may have been influenced by healthcare policy and a gender bias in recruitment. The National Health Service vascular check programme screens ${ }^{17}$ offers CVD risk assessment for age range of $40-75$ years and previous evidence ${ }^{18}$ have shown that women are more likely to join a general cardiovascular disease screening programme than men. Finally, the follow-up period of 6-12 months may have been too short for the outcome of specialist referral to be fed back into a patient's GP records.

\section{Clinical implications}

Patients with definite $\mathrm{FH}$ have been identified from this study and are now benefiting from treatment to reduce their greatly elevated risk of premature CHD. Further, this allows for cascade screening of additional family members from definite cases of $\mathrm{FH}$ in secondary care, an approach that is known to be costeffective. $^{19-22}$

\section{Future research}

This intervention, to more systematically identify patients with familial hypercholesterolaemia, is feasible to implement in GP, and facilitates successful recruitment of patients with raised cholesterol for appropriate assessment and referral to secondary care. Opportunistic recruitment may be improved in future studies by researchers more actively engaging with GP administrative and clinical staff, and by seeking to enhance effective implementation strategies, ${ }^{23}$ such as ensuring that study packs are available in all consultation rooms and repeated in-practice audit and feedback on the benefits of FH identification. To improve future implementation, we are currently assessing the post-study changes in practitioner behaviour on relevant clinical outcomes, such as family history and diagnosis of secondary causes, as well as the qualitative experience of those patients and healthcare professionals who participated in this study, including patients who declined follow-up. ${ }^{24}$

Using current (Simon-Broome) criteria, a large proportion of patients with possible $\mathrm{FH}$ are identified but not confirmed to have $\mathrm{FH}$ on specialist assessment. More resource-effective approaches to identify FH need to be developed. ${ }^{25-28}$ For instance, our recently developed approach (Familial Hypercholesterolaemia Case Acertainment Tool $)^{27}$ from routinely available data, held in primary care EHRs, takes into account patients already on statins, secondary causes of raised cholesterol, triglycerides and premature CHD when identifying patients who may have $\mathrm{FH}$.

The parameters derived in this study on eligibility, recruitment and diagnosis will directly inform a future cluster RCT in primary care. In any future trial, diagnosis of FH should be based on both clinical assessment and genetic testing. For a future trial in a large number of GPs, manual computer data extraction would likely not be feasible. Extraction of pseudoanonymised data from EHRs demonstrated in this and other related studies $^{29}$ can rapidly capture key trial outcome measures without burdening patients, for example, with forms seeking detailed information which may reduce response rates. ${ }^{30}$ Nevertheless, coded (automated) data extraction needs to be improved, for example, by incorporating computer templates to collate process data and outcomes of referral. Other outcomes measures with low levels of recording, or not routinely assessed in primary care, such as detailed family histories and physical signs of FH (xanthalasma and arcus senilus), need to be supplemented by additional clinician training and facilitated data capture using validated questionnaires. Any future robust trial will need to adopt a clusterrandomised design with a follow-up duration of at least 12 months. The trial sample size should also take account of patients already on statins with pre-treatment cholesterol above the threshold.

Acknowledgements The authors would like to thank other members of the FAMCHOL study steering group (Professor Carol Coupland, Professor Steve 
Humphries, Professor Denise Kendrick, Dr Daniel Lasserson, Professor Elizabeth Murray, Professor Andrew Neil, Mr Phil Rowlands), and Mr Tim Morell and Dr John Robinson (PRIMIS) for their technical support in extracting electronic health data from general practices.

Contributors The study was conceived and designed by NQ and JK, with input from the FAMCHOL study team. NQ and JT recruited, visited and collected the data from the patients and General Practice. AE-K collated data from secondary care. SW, NQ, JT conducted the study analysis of the primary and secondary outcome measures. The primary author NQ wrote the first draft of the manuscript, and the subsequent authors (SW, JT, JK) contributed towards revision and preparation of the final manuscript. All study authors have met authorship criteria set by International Committee of Medical Journal Editors (ICMJE).

Funding This work was funded by the National Institute for Health Research School of Primary Care Research (NIHR-SPCR) (FR2-95).

Competing interests NQ was a member of the NICE familial hypercholesterolaemia guideline development group (CG71).

\section{Patient consent Obtained.}

Ethics approval The feasibility study was approved by NRES Committee West Midlands-Solihull (Reference 12/WM/0322).

Provenance and peer review Not commissioned; externally peer reviewed.

Data sharing statement No additional data are available.

Open Access This is an Open Access article distributed in accordance with the terms of the Creative Commons Attribution (CC BY 4.0) license, which permits others to distribute, remix, adapt and build upon this work, for commercial use, provided the original work is properly cited. See: http:// creativecommons.org/licenses/by/4.0/

\section{REFERENCES}

1. Nordestgaard BG, Chapman MJ, Humphries SE, et al. Familial hypercholesterolaemia is underdiagnosed and undertreated in the general population: guidance for clinicians to prevent coronary heart disease: consensus statement of the European Atherosclerosis Society. Eur Heart J 2013;34:3478-90a.

2. [No authors listed]. Risk of fatal coronary heart disease in familial hypercholesterolaemia. Scientific Steering Committee on behalf of the Simon Broome Register Group. BMJ 1991;303:893-6.

3. [No authors listed]. Mortality in treated heterozygous familial hypercholesterolaemia: implications for clinical management. Scientific Steering Committee on behalf of the Simon Broome Register Group. Atherosclerosis 1999;142:105-12.

4. Neil A, Cooper J, Betteridge J, et al. Reductions in all-cause, cancer, and coronary mortality in statin-treated patients with heterozygous familial hypercholesterolaemia: a prospective registry study. Eur Heart J 2008;29:2625-33.

5. Neil HA, Hammond T, Huxley R, et al. Extent of underdiagnosis of familial hypercholesterolaemia in routine practice: prospective registry study. BMJ 2000;321:148.

6. Qureshi N, Humphries SE, Seed M, et al. Identification and management of familial hypercholesterolaemia: what does it mean to primary care? Br J Gen Pract 2009;59:773-6.

7. National Institute for Health and Care Excellence. Identification and management of familial hypercholesterolaemia (CG71). 2008. https://www.nice.org.uk/guidance/cg71.

8. Dhiman $\mathrm{P}$, Kai J, Horsfall L, et al. Availability and quality of coronary heart disease family history in primary care medical records: implications for cardiovascular risk assessment. PLOS ONE 2014;9: e81998.

9. Qureshi N, Armstrong S, Dhiman P, et al. Effect of adding systematic family history enquiry to cardiovascular disease risk assessment in primary care: a matched-pair, cluster randomized trial. Ann Intern Med 2012;156:253-62.

10. Qureshi N, Armstrong S, Saukko P, et al. Realising the potential of the family history in risk assessment and primary prevention of coronary heart disease in primary care: ADDFAM study protocol. BMC Health Serv Res 2009;9:184.

11. Hall R, Saukko PM, Evans $\mathrm{PH}$, et al. Assessing family history of heart disease in primary care consultations: a qualitative study. Fam Pract 2007;24:435-42.

12. Qureshi N, Bethea J, Modell B, et al. Collecting genetic information in primary care: evaluating a new family history tool. Fam Pract 2005;22:663-9.

13. Campbell NC, Murray E, Darbyshire J, et al. Designing and evaluating complex interventions to improve health care. BMJ 2007:334:455-9.

14. Gray J, Jaiyeola A, Whiting $M$, et al. Identifying patients with familial hypercholesterolaemia in primary care: an informatics-based approach in one primary care centre. Heart 2008;94:754-8.

15. Green P, Neely D, Humphries SE, et al. Improving detection of familial hypercholesterolaemia in primary care using electronic audit and nurse-led clinics. J Eval Clin Pract 2016;22:341-8.

16. Law MR, Wald NJ, Rudnicka AR. Quantifying effect of statins on low density lipoprotein cholesterol, ischaemic heart disease, and stroke: systematic review and meta-analysis. BMJ 2003;326:1423.

17. Department of Health. Putting prevention first-vascular checks: risk assessment and management. London, UK, 2008. http://www.dh. gov.uk/en/Publicationsandstatistics/Publications/PublicationsPolicy AndGuidance/DH_090277

18. Neil HA, Perera R, Armitage JM, et al. Estimated 10-year cardiovascular risk in a British population: results of a national screening project. Int J Clin Pract 2008;62:1322-31.

19. Marks D, Wonderling D, Thorogood M, et al. Cost effectiveness analysis of different approaches of screening for familial hypercholesterolaemia. BMJ 2002;324:1303.

20. Nherera L, Calvert NW, Demott K, et al. Cost-effectiveness analysis of the use of a high-intensity statin compared to a low-intensity statin in the management of patients with familial hypercholesterolaemia. Curr Med Res Opin 2010;26:529-36.

21. Pears R, Griffin M, Futema M, et al. Improving the cost-effectiveness equation of cascade testing for familial hypercholesterolaemia. Curr Opin Lipidol 2015;26:162-8.

22. Pears $\mathrm{R}$, Griffin M, Watson $\mathrm{M}$, et al. The reduced cost of providing a nationally recognised service for familial hypercholesterolaemia. Open Heart 2014;1:e000015.

23. Grimshaw JM, Thomas RE, MacLennan G, et al. Effectiveness and efficiency of guideline dissemination and implementation strategies. Health Technol Assess 2004;8(6):iii-iv.1-72.

24. Tranter J, Kai J, Qureshi N. Early identification of familial hypercholesterolaemia in general practice using patient-specific reminders: focus group with General Practitioners. BMC Health Services Research 2014;14(Suppl 2):P97.

25. Bell DA, Edwards G, Hooper AJ, et al. The potential role of an expert computer system to augment the opportunistic detection of individuals with familial hypercholesterolaemia from a community laboratory. Clinical Chimica Acta 2015;448:18-21.

26. Bell DA, Watts GF. Screening for familial hypercholesterolemia: primary care applications. Clin Lipidol 2015;10:295-8.

27. Weng SF, Kai J, Andrew Neil H, et al. Improving identification of familial hypercholesterolaemia in primary care: Derivation and validation of the familial hypercholesterolaemia case ascertainment tool (FAMCAT). Atherosclerosis 2015;238:336-43.

28. Kirke AB, Barbour RA, Burrows $S$, et al. Systematic detection of familial hypercholesterolaemia in primary health care: a community based prospective study of three methods. Heart Lung Circ 2015;24:250-6.

29. Troeung L, Arnold-Reed D, Chan She Ping-Delfos W, et al. A new electronic screening tool for identifying risk of familial hypercholesterolaemia in general practice. Heart 2016;102:855-61.

30. Pit SW, Vo T, Pyakurel S. The effectiveness of recruitment strategies on general practitioner's survey response rates-a systematic review. BMC Med Res Methodol 2014;14:1. 


\section{Correction: Feasibility of improving identification of familial hypercholesterolaemia in general practice: intervention development study}

Qureshi N, Weng S, Tranter J, et al. Feasibility of improving identification of familial hypercholesterolaemia in general practice: intervention development study. BMJ Open 2016;6: e011734.

The following acknowledgement should be included in this paper:

This paper presents independent research funded by the National Institute for Health Research School for Primary Care Research (NIHR SPCR).

In addition, the following disclaimer should be included in this paper:

The views expressed are those of the author(s) and not necessarily those of the NIHR, the NHS or the Department of Health.

Open Access This is an Open Access article distributed in accordance with the terms of the Creative Commons Attribution (CC BY 4.0) license, which permits others to distribute, remix, adapt and build upon this work, for commercial use, provided the original work is properly cited. See: http://creativecommons.org/licenses/by/4.0/

BMJ Open 2016;6:e011734corr1. doi:10.1136/bmjopen-2016-011734corr1 